\title{
Tunable multi-band chiral metamaterials based on double-layered asymmetric split ring resonators
}

\author{
Xiuli Jia ${ }^{\mathrm{a}}$, Xiaoou Wanga,*, Qingxin Meng ${ }^{\mathrm{a}}$, Zhongxiang Zhou ${ }^{\mathrm{a}}$ \\ ${ }^{a}$ Harbin institute of technology, Harbin 150001, China
}

\begin{abstract}
We have numerically demonstrated chiral metamaterials based on double-layered asymmetric Au film with hollow out design of split ring resonators on either side of the polyimide. Multiple electric dipoles and magnetic dipoles resulted from parallel and antiparallel currents between the eight split ring resonators. Multiband circular dichroism is found in the visible frequency regime by studying the transmission properties. Huge optical activity and the induced multi-band negative refractive index are obtained at resonance by calculating the optical activity and ellipticity of the transmitted E-fields. Chirality parameter and effective refractive index are retrieved to illustrate the tunable optical properties of the metamaterials. The underlying mechanisms for the observed circular dichroism are analyzed. These metamaterials would offer flexible electromagnetic applications in the infrared and visible regime.
\end{abstract}

Keywords: chiral metamaterials, circular dichroism, optical activity, negative refractive index, electric dipoles, magnetic dipoles

2010 MSC: 00-01, 99-00

\section{Introduction}

Metamaterials with negative refraction have drawn significant attention since Smith et al. successfully prepared such metamaterials in the laboratory

\footnotetext{
National Natural Science Foundation of China (No 61205011).

* Corresponding author

Email address: wxo@hit.edu.cn (Xiaoou Wang)
} 
in 2000 Ref. 11. Recently, increasing attention has been focused on chiral 5 metamaterials (CMMs) owing to their attractive electromagnetic (EM) properties, such as their large optical activity, strong circular dichroism (CD) effect, negative refractive index (NRI), and asymmetric transmission effect Ref.

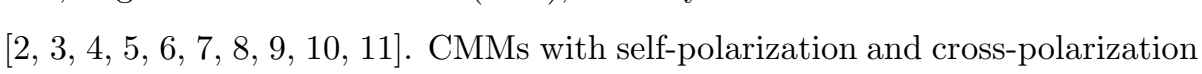
between electromagnetic fields can achieve NRI depending on their chirality, whether or not the permittivity and permeability are negative. Furthermore, they can exhibit strong optical activity and lower loss of the electromagnetic wave (EMW) transmission Ref. [3].

Recently, negative refraction CMMs have shown large optical activities (e.g., e.g., $\left.\theta=130^{\circ}\right)$ Ref. [12], high-frequency regions $\left(3 \times 10^{14} \mathrm{~Hz}\right)$ Ref. [13], lower loss

$15 \quad(F O M=4.2)$ Ref. [14], and high negative refraction value $(\mathrm{n}=-180)$ Ref. [15]. Chirality is the lack of the internal mirror symmetry of a molecule or artificial structure. Optical activity is a major characteristic of CMMs. In nature, optical activity is caused by the intrinsic spiral feature of chiral molecules or the spiral arrangement of its atomic molecules. The optical activity of a natural chiral medium is caused by the intrinsic helical characteristics of molecules or the spiral alignment of atoms, and the root of the rotation is circular birefringence, and strength is very weak. For artificial CMMs, optical activity is caused by the optical spatial dispersion of structural chirality. Compared with nature, CMMs have higher optical activities and chiralities Ref. [3, 12, 16, 17].

CD $(\Delta)$ refers to one of the main physical parameters for characterizing the absorption of the RCP wave (+, right circular polarization wave) and the LCP wave (-, left circular polarization wave):

$$
\Delta=\left|T_{++}\right|-\left|T_{--}\right|
$$

The polarization azimuthal rotation angle $(\theta)$ refers to the angle of rotation with respect to the plane of polarization:

$$
\theta=\frac{\arg \left(T_{--}\right)-\arg \left(T_{++}\right)}{2}
$$



and incident waves, and also measures the CD effect. It is caused by electromagnetic coupling between two layers of the CMM:

$$
\eta=\frac{1}{2} \arctan \left(\frac{\left|T_{++}\right|^{2}-\left|T_{--}\right|^{2}}{\left|T_{++}\right|^{2}+\left|T_{--}\right|^{2}}\right)
$$

Here, $T_{++}$refers to the transmission coefficient of the RCP wave and $T_{--}$ refers to the transmission coefficient of the LCP wave. Using the retrieval meth35 ods for CMMs Ref. [18], we can obtain the effective impedance $\left(Z_{\text {eff }}\right)$, the refractive index of the RCP and LCP waves $\left(n_{ \pm}\right)$, and the effective refractive index $\left(n_{e f f}\right)$ of the chiral medium:

$$
\begin{gathered}
Z_{\text {eff }}=\sqrt{\frac{(1+R)^{2}-T_{++} T_{--}}{(1-R)^{2}-T_{++} T_{--}}} \\
n_{ \pm}=\frac{-j}{k_{0} d} \ln \left[\frac{1}{T \pm \pm}\left(1-\frac{Z_{e f f}-1}{Z_{e f f}+1} R\right)\right] \\
n_{e f f}=\left(n_{+}+n_{-}\right) / 2
\end{gathered}
$$

Here, $\kappa_{0}$ refers to the wave number in free space, and $d$ refers to the equivalent thickness of the chiral structure. The effective impedance and equivalent 40 refractive index are the basis for obtaining the effective chiral parameter $\left(\kappa_{e f f}\right)$ and effective permittivity $\left(\varepsilon_{e f f}\right)$ :

$$
\kappa_{e f f}=\left(n_{+}-n_{-}\right) / 2 \text {. }
$$

$$
\varepsilon_{e f f}=n_{e f f} / Z_{e f f} .
$$


In this work, we used the finite difference time domain (FDTD) method to systematically study the complementary split ring shaped CMM. In this context, it has been shown that an NRI and a large optical activity occur at infrared and

45 visible light frequencies. The geometrical parameters are examined to explore the strong optical activity, CD effect, and NRI at these frequencies. Endowed with these rich EM (optical) properties, CMMs may lead to many applications in photonic devices on account of their strong optical activity (polarization rotation effect) and $\mathrm{CD}$ effect.

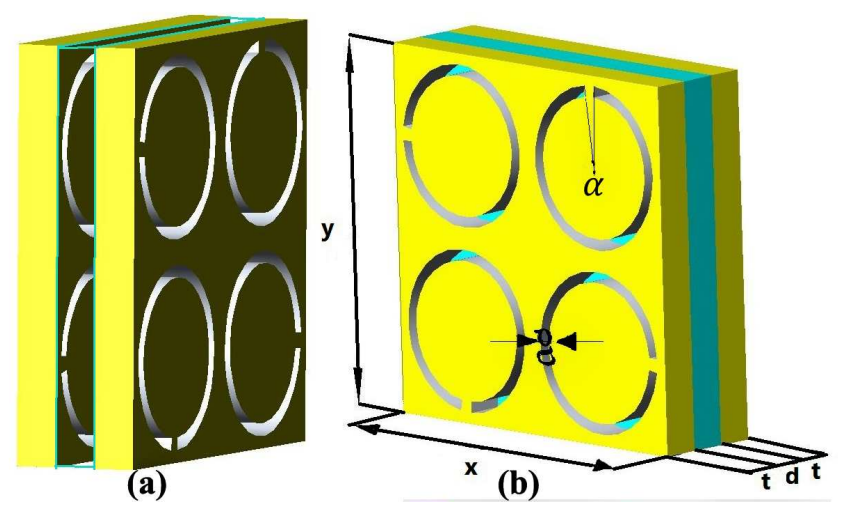

Fig. 1. The unit cell of the chiral metamaterial (CMM): (a) Perspective view of the structure includes hollowed-out split ring resonators in front and back. (b) Full dimensions of the three-layered structure composed of $\mathrm{Au}$ layer, polyimide layer and Au layer another, $\mathrm{x}=\mathrm{y}=500 \mathrm{~nm}$, the metal layer thickness is represented by $t$, the dielectric layer thickness is represented by $d$, the width of the rings is represented by $g$ (between outer radius and minus inner radius), and the angle of splits is represented by $\alpha$.

Babinet's principle was applied to the design of the metamaterial, resulting in both a complementary spectral response and fields Ref. [19, 20, 21, 22]. We created a chiral structure in an array of conjugated bilayer metal split ring 
resonators, as shown in Fig. 1 (b), where the conjugated bilayer metal split ring resonator is depicted as yellow, and the dielectric layer is depicted as blue. The unit size of the chiral structure is $\mathrm{x}=\mathrm{y}=500 \mathrm{~nm}$. The CMM consists of three layers (i.e., Au/polyimide/Au). The metal layer thickness is represented by $t$, the dielectric layer thickness by $d$, the width of the ring by $g$ (outer radius minus inner radius), and the angle of cut by $\alpha$. The location of the cut rotates by $90^{\circ}$ from ring to ring. The dielectric constant of the polyimide is 3.5 , and the loss tangent is $\tan \tan \delta=0.003$. Gold can be described by Drude dispersion model as follows: $\varepsilon=1-\omega_{p} / \omega\left(\omega+i \omega_{\gamma}\right)$. The plasma frequency is $\omega_{p}=1.37 \times 10^{16}$ $\mathrm{s}^{-1}$, and the collision frequency is $\omega_{\gamma}=2.04 \times 10^{14} \mathrm{~s}^{-1}$ Ref. [23].

The current density distributions and electric field distributions were obtained from numerical simulations at nine resonance frequencies where strong optical activity occurs, as shown by Fig. 2 .

For the multi-layer chiral structure, strong coupling was found between each layer of metal, which formed symmetric and asymmetric current distributions on the surface of the metals. This also generated a strong coupling between the electric and magnetic field, as well as a large chiral parameter, thereby achieving negative refraction. From the rings on the metal surfaces in the unit structure, multiple current density distribution areas are located inside and outside of the split rings, which can be divided into two regions: the parallel direction region, forming an electric dipole, and the anti-parallel direction region, forming a magnetic dipole, as shown in Fig. 2 (a). This indicates that the coupling method of the rings with the EMW is both electric and magnetic in nature. The structure we designed forms multiple electric dipoles and magnetic dipoles, indicating that the couplings between this chiral structure and EMW are multiple electric and magnetic couplings. This mechanism of resonance is different

80 from that of the single electric and magnetic dipoles of the ${ }^{\prime}{ }^{\prime}{ }^{\prime}$ chiral structure Ref. 15, 24]. Thus, the nine resonance frequencies result from the joint action of multiple electric and magnetic dipoles. This proves that the shape of the structure directly affects the number of resonance frequencies and the broad of frequency bands. 
current density and electric field

(a)

(b)
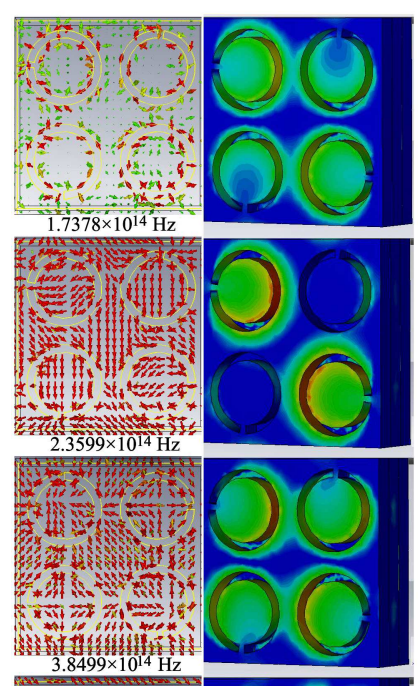

$3.8499 \times 10^{14} \mathrm{~Hz}$

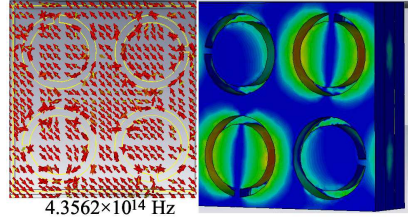

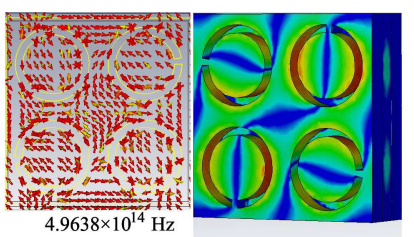

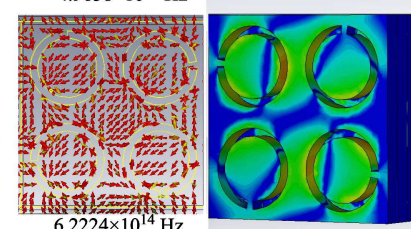

$6.2224 \times 10^{14} \mathrm{~Hz}$
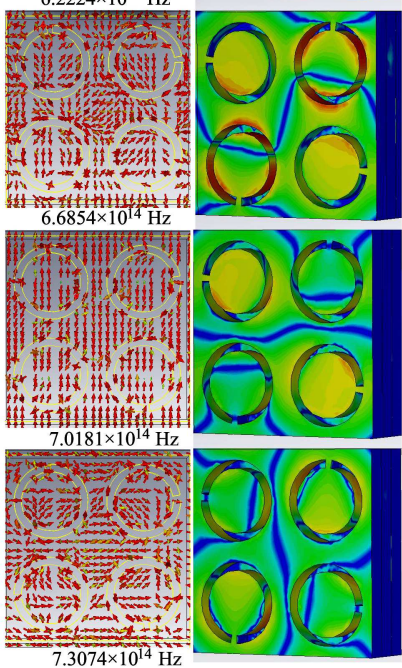

Fig. 2. The physical mechanism analysis of multiple electric dipoles and multiple magnetic dipoles: (a) Distributions of the surface current density of nine resonance frequencies. (b) Electric field of nine resonance frequencies.

To gain a better understanding of the multiple resonance frequency mechanism, we also provide the calculated electric field distributions in Fig. 2. As shown in Fig. 2 (b), the electric field strength increases with the increase in the resonance frequency. Through an analysis of the electric field under multiple resonance frequencies, we believe that there are two main reasons for this: 1)

90 the CMM structure unit itself produces different resonance frequencies, 2) with the increase of the resonance frequency, the interaction increases between the adjacent units. From the electric field distribution in Fig. 2, we can see that for resonance frequencies under $6.2224 \times 10^{14} \mathrm{~Hz}$ (including $6.2224 \times 10^{14} \mathrm{~Hz}$ ), 
the electric field forms in the structure unit and the interaction between the adjacent units are weak. However, at higher resonance frequencies, we find that the interaction between the adjacent structural units is greatly enhanced.

\section{SIMULATION RESULTS AND DISCUSSION}

\subsection{Optimization of $C M M^{\prime} s$ structure}

According to the effective media theory, the effective EM parameters could be retrieved from the complex scattering parameters (S-parameters, include transmission $T$ and refraction coefficients $R$ ). The simulation has been performed based on the standard finite difference time domain (FDTD) method. The periodic boundary conditions are applied to the $\mathrm{X}$ and $\mathrm{Y}$ directions and the absorbing boundary conditions to the $\mathrm{Z}$ direction as well. Based on above numerical simulation method, the final calculated S-parameters of multiple samples as shown by Fig. 3 (a) - (c).
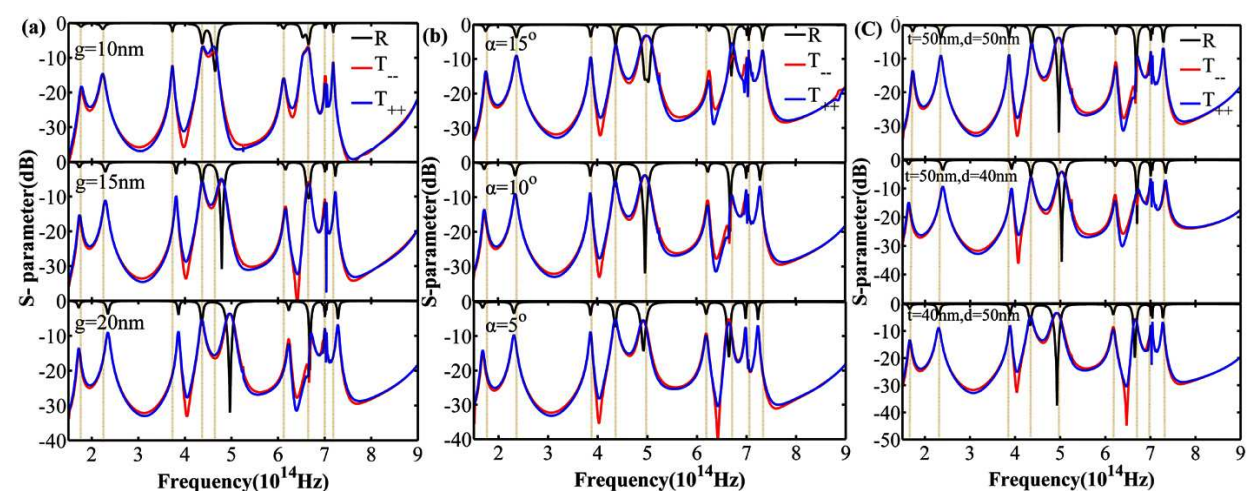

Fig. 3. S-parameters of multiple samples: (a) $\alpha=10^{\circ}, g=10 \mathrm{~nm}, 15 \mathrm{~nm}$ and $20 \mathrm{~nm}$. (b) $g=20 \mathrm{~nm}, \alpha=15^{\circ}, 10^{\circ}$ and $5^{\circ}$. (c) $\alpha=10^{\circ}, g=20 \mathrm{~nm}, t$ $=50 \mathrm{~nm}, d=50 \mathrm{~nm}, \alpha=10^{\circ}$ and $g=20 \mathrm{~nm}, t=50 \mathrm{~nm}, d=40 \mathrm{~nm}$ and $\alpha$ $=10^{\circ}, g=20 \mathrm{~nm}, t=40 \mathrm{~nm}$ and $d=50 \mathrm{~nm}$.

For the purpose of comparison, in Fig. 3 (a) $\alpha$ remains constant at $10^{\circ}$ while $g$ is adjusted. The CMM has nine resonance frequencies (transmission 
peaks shown by yellow lines). This indicates that the resonance frequency regions will blue-shift with the increase in $g$, and that the reflection coefficient $R$ decreases significantly with the increase of $g$ at about $5 \times 10^{14} \mathrm{~Hz}$ and $6.5 \times 10^{14}$ Hz. The reflection coefficient $R$ and transmission coefficients $T_{++} / T_{--}$show that when the $R$ is at its minimum, $T_{++} / T_{--}$reach their maxima. Furthermore, the bands around resonance frequencies become wider with the increase in $g$, which results in superior transmission for the visible light region. Under the impact of chirality, a difference exists between $T_{++}$and $T_{--}$, which indicates that the chiral structure causes different transmissions in the RCP and LCP waves. In particular, at about $4 \times 10^{14} \mathrm{~Hz}, T_{++}$is nearly $10 \mathrm{~dB}$ higher than $T_{--}$. Furthermore, $T_{++}$reversal smaller than $T_{--}$at about $6.3 \times 10^{14} \mathrm{~Hz}$ with the increase in $g=20 \mathrm{~nm}$. This sufficiently reflects the optical spatial dispersion of the CMMs.

For the purpose of comparison, in Fig. 3 (b) $g$ remains constant at $20 \mathrm{~nm}$ while $\alpha$ is adjusted. Upon the reduction of $\alpha$, the resonance frequencies were not found to generate movement (unlike the case of the increase in $g$ ), though the $R$ curve was found to increase. The structure was designed as a hollow structure. If the hollow area is large, EMW transmittance should be maximal and reflection should be minimal. For example, if $g$ is increased, then EMW transmittance is improved. However, when we reduce the cut angle $\alpha$ in order to further increase the size of the hollow area, we find that EMW transmittance is not improved. As shown in Fig. 3 (c), the $R$ curve is at a minimum and $T_{++} / T_{--}$are at their maxima at $4.9638 \times 10^{14} \mathrm{~Hz}$ and $6.6854 \times 10^{14} \mathrm{~Hz}$, respectively, when $g=20 \mathrm{~nm}$ and $\alpha=10$. This is because each ring can be regarded as an $L C$ oscillatory circuit, thereby forming an electric dipole for the transmission of electric energy, the coupling of adjacent rings forms a magnetic dipole for the transmission of magnetic energy. Under these conditions, the magnetic field energy is $L Q^{\prime 2} / 2$ and the electric field energy is $Q^{2} / 2 C$ Ref. [5]. When $\alpha$ and $g$ are increased, this makes the inductance $L$, capacitance $C$, and the electrical charge $Q$ all increase, which means that the magnetic field energy and electric field energy increase as well. The transmission energy is equal to the sum of the electric and magnetic 
${ }_{140}$ field energies, therefore, $T$ increases as $R$ decreases. This result is consistent with the results of the previously performed qualitative analysis. When $g$ and $\alpha$ are decreased, this makes $L$ decrease, while $C$ and $Q$ increase. Accordingly, the electric field energy increases but the magnetic field energy decreases, and the transmission potential increases non-monotonically. Therefore, when the ring cut $\alpha$ decreases, the transmission coefficient will increase initially and then later decrease.

By adjusting $g$ and $\alpha$, we found that $g$ determines the resonance frequencies and that the EMW transmittance increases with $g$. However, $\alpha$ does not affect the resonance frequencies, and affects the EMW transmittance only to a certain extent. In summary, the optimal parameters of this structure should be $g=20$ $\mathrm{nm}$ and $\alpha=10^{\circ}$.

While maintaining the parameters at $g=20 \mathrm{~nm}, \alpha=10$ (along with the size of the unit cell), the parameters $t$ and $d$ were then adjusted. We obtained S-parameters of multiple samples through the simulation for $t=50 \mathrm{~nm}$ and $d$ ${ }_{155}=50 \mathrm{~nm}, t=50 \mathrm{~nm}$ and $d=40 \mathrm{~nm}$, as well as $t=40 \mathrm{~nm}$ and $d=50 \mathrm{~nm}$, as shown in Fig. 3 (c). According to this figure, the dielectric medium thickness $d$ has no effect on the S-parameter, whereas the metal thickness $t$ has a significant effect on $T_{--}$in the high-frequency region. At $t=40 \mathrm{~nm}$, we can also see the decrease of $T_{--}$occur only in the high-frequency region $\left(6.465 \times 10^{14} \mathrm{~Hz}\right)$, $T_{--}$decreases nearly $20 \mathrm{~dB}$, but $T_{++}$remain unchanged, which illustrates an increase in circular dichroism. And in lower frequency $\left(4.035 \times 10^{14} \mathrm{~Hz}\right) T_{--}$ remain the value with the other samples. That is because of the influence of the metal skin depth in high-frequency regions. Furthermore, in high-frequency regions, the metal's absorption of EMWs is influenced by the metal size: smaller values of $t$ lead to a higher absorption of EMWs.

\subsection{Results and discussion}

On the basis of Eqs. (1)-(3), $\Delta, \theta$, and $\eta$ are shown in Fig. 4 (a) and (b). As shown in Fig. 4 (a), $\alpha$ remains unchanged at $10^{\circ}$, while $g$ is adjusted. In Fig. 4 (b), $g$ remains unchanged at $20 \mathrm{~nm}$ while $\alpha$ is adjusted. On the basis of 
the increase $g$ and $\alpha$, the resonance region around $5 \times 10^{14} \mathrm{~Hz}$ move to blue-shift obviously. A significant increase in $\mathrm{CD}(\Delta)$ indicates a big difference between transmission spectra, and two significant peaks. This result indicates that the chiral structure has good CD in the visible light region, and that the increase in ring width $g$ and ring cut $\alpha$ can improve the CD of the CMM.

$\theta$ refers to the polarized angle of rotation of the exiting wave polarization plane against the incident wave per unit length. When $g=10 \mathrm{~nm}, \alpha=10$ and $g=20 \mathrm{~nm}, \alpha=15^{\circ}$ (decrease hollow area), $\theta$ is up to maximal value of $180^{\circ}$, higher than that of other chiral negative refraction metamaterials in infrared and visible light regions Ref. 5]. Therefore, the proposed structure has improved optical activity.

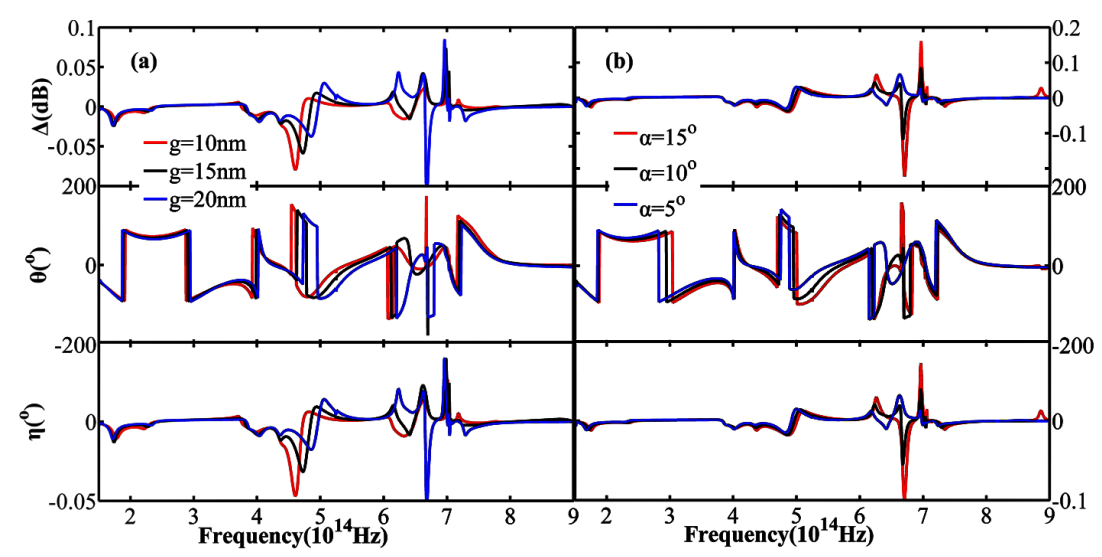

Fig. 4. Optical properties of multiple samples: (a) $\alpha=10^{\circ}, g=10 \mathrm{~nm}, 15$ $\mathrm{nm}$ and $20 \mathrm{~nm}$. (b) $g=20 \mathrm{~nm}, \alpha=15^{\circ}, 10^{\circ}$ and $=5^{\circ}$.

$\eta$ refers to the change in the angle of the exiting wave against the polarization of the incident wave, as shown in Fig. 4 (a) and (b). These results indicate that the polarization of the incident wave of the CMM has changed at the points of resonance frequency. The regulation of the chiral structure parameters on $\eta$ is similar to their regulation on $\Delta . \eta$ is significantly small throughout the whole frequency range (in most of the frequency regions, $\eta$ is zero). This illustrates 
the extremely small ellipticity of the transmitted wave, which can be used to minimize the polarization distortion and achieve high transmission. In this way, ring

As shown by the $n_{+}$in Figs. 5 (a) and (b), the chiral structure has five negative refraction bands in the simulation frequency range of $1.5 \times 10^{14} \mathrm{~Hz}$ to 215 range. Similarly, for $g=20 \mathrm{~nm}$ and $\alpha=10$, the negative refractive index frequencies are as follows: $1.515 \times 10^{14} \mathrm{~Hz}$ to $1.785 \times 10^{14} \mathrm{~Hz}, 3.1425 \times 10^{14} \mathrm{~Hz}$ to $3.87 \times 10^{14} \mathrm{~Hz}, 5.1825 \times 10^{14} \mathrm{~Hz}$ to $5.58 \times 10^{14} \mathrm{~Hz}, 6.30 \times 10^{14} \mathrm{~Hz}$ to $6.315 \times 10^{14}$ 
$\mathrm{Hz}$, and $6.6975 \times 10^{14} \mathrm{~Hz}$ to $7.17 \times 10^{14} \mathrm{~Hz}$, accounting for $26 \%$ of the total 220 stimulation frequency range and for $25 \%$ of the visible light frequency region. The extreme value of $n_{+}$is -1.9 .

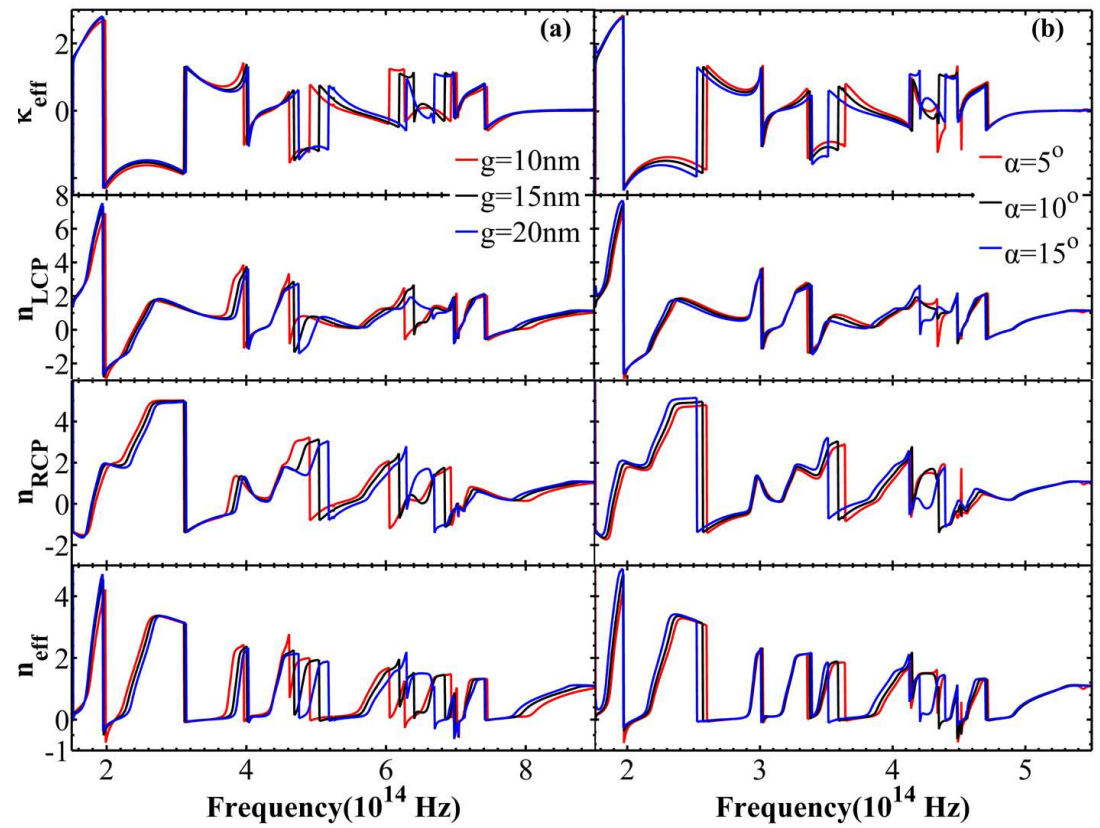

Fig. 5. Chiral parameter and negative refractive index of multiple samples: (a) $\alpha=10^{\circ}, g=10 \mathrm{~nm}, 15 \mathrm{~nm}$ and $20 \mathrm{~nm}$. (b) $g=20 \mathrm{~nm}, \alpha=15^{\circ}, 10^{\circ}$ and $5^{\circ}$.

As shown in Figs. 5 (a) and (b), $n_{\text {eff }}$ has nine equivalent negative refractive index bands, among which there are eight broad negative refractive index bands, as follows: $1.95 \times 10^{14} 4 \mathrm{~Hz}$ to $2.1375 \times 10^{14} \mathrm{~Hz}, 3.1425 \times 10^{14} \mathrm{~Hz}$ to $3.4575 \times 10^{14}$ $\mathrm{Hz}, 4.03 .5 \times 10^{14} \mathrm{~Hz}$ to $4.05 \times 10^{14} \mathrm{~Hz}, 5.1825 \times 10^{14} \mathrm{~Hz}$ to $5.235 \times 10^{14} \mathrm{~Hz}, 5.25 \times 10^{14}$ $\mathrm{Hz}$ to $5.28 \times 10^{14} \mathrm{~Hz}, 6.69 \times 10^{14} 4 \mathrm{~Hz}$ to $6.8325 \times 10^{14} \mathrm{~Hz}, 6.975 \times 10^{14} \mathrm{~Hz}$ to $7.08 \times 10^{14} \mathrm{~Hz}$, and $7.425 \times 10^{14} \mathrm{~Hz}$ to $7.4925 \times 10^{14} \mathrm{~Hz}$, accounting for $14 \%$ of the total stimulation frequency range and for $12 \%$ of the visible light frequency range. The number and width of frequency bands of this negative refraction 
CMM in the visible light region are far better than those of the fish-net structure negative refractive index material discussed in the literature Ref. [25].

As shown in Fig. 5, the change in structural parameters has a regulating effect on the negative refraction, wherein the resonance frequencies blue-shift with increases in the ring width $g$ and ring cut $\alpha$.

A longitudinal comparison of $\kappa_{e f f}, n_{-}, n_{+}$and $n_{e f f}$ shows that $n_{-}$appears negative for the negative minimum value of the chiral parameter, and continues negative in a certain range that the chiral parameter maintains lower value of the chiral parameter. $n_{+}$appears in the negative maximum value of the chiral parameter, and continues negative in a certain range that the chiral parameter maintains higher value of the chiral parameter. These results indicate that the chiral structure has a promoting effect and an inhibiting effect on the LCP and RCP waves, respectively. $n_{\text {eff }}$ is represented in a certain extreme value that the chiral parameter maintains, whether it is negative or not further depends on the values of $n_{-}$and $n_{+}$.

\subsection{The discussion of loss}

The loss at $4.925 \times 10^{14} \mathrm{~Hz}, 5.0625 \times 10^{14} \mathrm{~Hz}$ and $6.6875 \times 10^{14} \mathrm{~Hz}$ have nearly complete loss of $100 \%$ in this chiral metamaterial based on double-layered asymmetric split ring resonators as shown in Fig. 6 (a). But the loss is low at

250 the bands of negative refractive index, where the negative of $n_{-}$at $1.95 \times 10^{14}$ $\mathrm{Hz}$ to $2.4675 \times 10^{14} \mathrm{~Hz}, 4.035 \times 10^{14} \mathrm{~Hz}$ to $4.1775 \times 10^{14} \mathrm{~Hz}, 4.755 \times 10^{14} \mathrm{~Hz}$ to $4.965 \times 10^{14} \mathrm{~Hz}, 6.975 \times 10^{14} \mathrm{~Hz}$ to $7.035 \times 10^{14} \mathrm{~Hz}, 7.425 \times 10^{14} \mathrm{~Hz}$ to $7.785 \times 10^{14}$ $\mathrm{Hz}$ and the negative of $n_{+}$at $1.515 \times 10^{14} \mathrm{~Hz}$ to $1.785 \times 10^{14} \mathrm{~Hz}, 3.1425 \times 10^{14} \mathrm{~Hz}$ to $3.87 \times 10^{14} \mathrm{~Hz}, 5.1825 \times 10^{14} \mathrm{~Hz}$ to $5.58 \times 10^{14} \mathrm{~Hz}, 6.30 \times 10^{14} \mathrm{~Hz}$ to $6.315 \times 10^{14}$ ${ }_{255} \mathrm{~Hz}, 6.6975 \times 10^{14} \mathrm{~Hz}$ to $7.17 \times 10^{14} \mathrm{~Hz}$. The loss of metamaterials from both electrical resonance and magnetic resonance is very large usually, but chiral metamaterials can achieve negative refractive index by increasing the effective chiral parameters $\left(\kappa_{e f f}\right)$ as shown in Fig. 5 and decreasing effective permittivity $\left(\varepsilon_{e f f}\right)$ as shown in Fig. $6(\mathrm{~b})$, this method does not need to magnetic resonance, 260 therefore the chiral metamaterials have lower loss. 

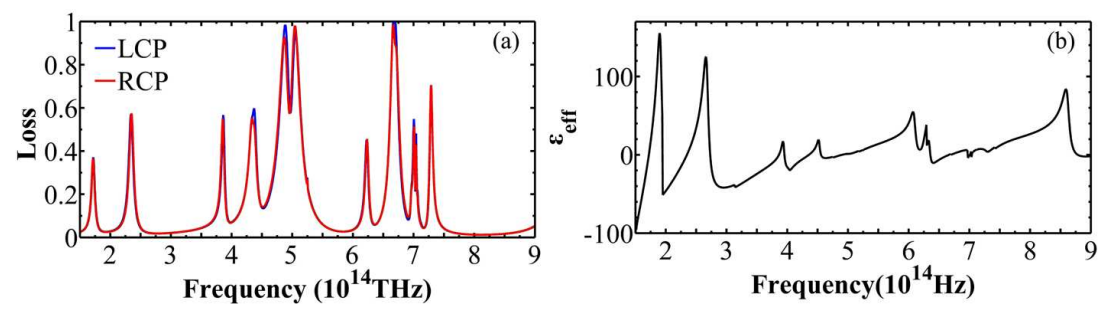

Fig. 6. The structural parameters of $g=20 \mathrm{~nm}, \alpha=10^{\circ}, t=50 \mathrm{~nm}, d=$ 50nm: (a) The loss of the structure. (b) The effective permittivity $\varepsilon_{\text {eff }}$ of the structure.

\section{CONCLUSIONS}

In summary, we have designed and optimized a chiral metamaterial based on double-layered asymmetric split ring resonators in the infrared and visible light regions. The S-parameter obtained in the frequencies of $1.5-910 \times 10^{14} \mathrm{~Hz}$ has nine resonance regions. The retrieval calculation shows that the LCP and $\mathrm{RCP}$ waves in this frequency range have five negative refractive index frequency bands and eight broad equivalent negative refraction frequency bands, respectively, with a higher and wider frequency range than the CMMs in the existing literature Ref. 25]. With regard to the visible light range, the negative refraction bands of the LCP wave, the RCP wave, and the equivalent account for $22 \%$, $25 \%$, and $12 \%$ of total visible light region, respectively. Meanwhile, the number of negative refraction bands is more than that reported in the literature Ref. [15, 24]. By increasing the ring width $g$ and ring cut $\alpha$ the resonance frequencies can be regulated, thereby optimizing the negative refraction and optical activity are obtained. We believe that $g=20 \mathrm{~nm}$ and $\alpha=10^{\circ}$ represent the best parameters for this CMM. This CMM has a large optical activity, and a high angle of rotation (i.e., up to $180^{\circ}$ ). We also analyzed the resonance mechanism of this chiral structure for achieving eight bands of negative refraction. We believe that the joint action of so many electric and magnetic dipoles is an important factor for achieving multi-band negative refraction. We believe that this CMM will be significantly applied in functional materials, optical devices, and other fields. 


\section{ACKNOWLEDGMENTS}

This work was supported by the National Natural Science Foundation of China (No 61205011)and the National Science Foundation of China (NSFC) (No. 61205093).

\section{References}

[1] D. R. Smith, W. J. Padilla, D. C. Vier, S. C. Nemat-Nasser, S. Schultz, Composite medium with simultaneously negative permeability and permittivity, Appl. Phys. Lett 84 (2000) 4184.

[2] S. Tretyakov, I. Nefedov, A. Sihvola, S. Maslovski, C. Simovski, Waves and energy in chiral nihility, J. Electromagn. Waves Appl. 17 (2003) 695.

[3] J. B. Pendry, A chiral route to negative refraction, Science 306. (2004) 1353.

[4] A. V. Rogacheva, V. A. Fedotov, A. S. Schwanecke, N. I. Zheludev, Giant gyrotropy due to electromagnetic-field coupling in a bilayered chiral structure, Phys. Rev. Lett. 97 (2006) 177401.

[5] H. Liu, D. A. Genov, D. M. Wu, Y. M. Liu, Z. W. Liu, C. Sun, S. N. Zhu, , X. Zhang, Magnetic plasmon hybridization and optical activity at optical frequencies, Phys. Rev. B 76 (2007) 073101.

[6] T. Q. Li, H. Liu, T. Li, S. M. Wang, F. M. Wang, R. X. Wu, P. Chen, S. N. Zhu, X. Zhang, Magnetic resonance hybridization and optical activity of microwaves in a chiral metamaterial, Appl. Phys. Lett. 92 (2008) 131111.

[7] M. Decker, S. Linden, M. Wegener, Coupling effects in low-symmetry planar split-ring resonator arrays, Opt. Lett. 34 (2009) 1579.

[8] B. Wang, T. Koschny, C. Soukoulis, Wide-angle and polarizationindependent chiral metamaterial absorber, Phys. Rev. B 033108 (2009) 80. 
[9] R. Singh, E. Plum, W. L. Zhang, N. I. Zheludev, Highly tunable optical activity in planar achiral terahertz metamaterials, Opt. Express 18 (2010) 13425.

[10] B. Wang, T. Koschny, M. Kafesaki, C. M. Soukoulis, Chiral metamaterials: Simulations and experiments 11 (2009) 114003.

[11] M. Mutlu, A. Akosman, A. Serebryannikov, E. Ozbay, Asymmetric transmission of linearly polarized waves and polarization angle dependent wave rotation using a chiral metamaterial, Opt. Express 19 (2011) 14290.

[12] J. Zhou, B. W. J. Dong, T. Koschny, M. Kafesaki, C. M. Soukoulis, Negative refractive index due to chirality, Phys. Rev. B 79 (2009) 121104.

[13] J. Dong, J. Zhou, T. Koschny, C. Soukoulis, Bi-layer crosschiralstructure with strong optical activity and negative refractive index, Opt.Express 17 (2009) 14172.

[14] K. Song, X. P. Zhao, Q. H. Fu, Y. H. Liu, W. R. Zhu, Wide-angle 90polarization rotator using chiral metamaterial with negative refractive index, J. Electromagnet. Wave 26 (2012) 1967.

[15] W. Panpradit, A. Sonsilphong, C. Soemphol, N. Wongkasem, High negative refractive index in chiral metamaterials, J. Opt. 14 (2012) 075101.

[16] S. Tretyakov, A. Sihvola, L. Jylhä, Backward-wave regime and negative refraction in chiral composites, Photon. Nanostruct. Fundam. Appl. 3 (2005) 107.

[17] E. Plum, J. Zhou, J. Dong, V. A. Fedotov, T. Koschny, C. M. Soukoulis, N. I. Zheludev, Metamaterial with negative index due to chirality, Phys. Rev. B 79 (2009) 035407.

[18] X. Chen, M. T. Grzegorczyk, B. Wu, J. Pacheco, Jr., J. Kong, Robust method to retrieve the constitutive effective parameters of metamaterials, Phys. Rev. B 70 (2004) 016608. 
[19] Z. Li, K. B. Alici, E. Colak, E. Ozbay, Complementary chiral metamaterials with giant optical activity and negative refractive index, Appl. Phys. Lett. 98 (2011) 161907.

[20] F. Falcone, T. L. Opetegi, M. A. G. Laso, J. D. Baena, J. Bonache, M. Beruete, R. R. Marques, F. F. Martn, M. Sorolla, Babinet principle applied to the design of metasurfaces and metamaterials, Phys. Rev. Lett. 93 (2004) 19740.

[21] T. Zentgraf, T. P. Meyrath, A. Seidel, S. Kaiser, H. Giessen, Babinet/s principle for optical frequency metamaterials and nanoantennas, Phys. Rev. B 76 (2007) 033407.

[22] N. Liu, S. Kaiser, H. Giessen, Magnetoinductive and electroinductive coupling in plasmonic metamaterial molecules, Adv. Mater. 20 (2008) 4521.

[23] S. Zhang, Y. S. Park, J. Li, X. Lu, W. Zhang, X. Zhang, Negative refractive index in chiral metamaterials, Phys. Rev. Lett. 102 (2009) 023901.

[24] A. Lakhtakia, V. V. Varadan, V. K. Varadan, Eigenmodes of a chiral sphere with a perfectly conducting coating, J. Opt. Soc. Am. A 5 (1988) 175.

[25] M. Choi, J. Choe, B. Kang, C. Choi, A flexible metamaterial with negative refractive index at visible wavelength, Curr. Appl. Phys. 13 (2013) 1723. 